\title{
Targeting KCa1.1 Channels with a Scorpion Venom Peptide for the Therapy of Rat Models of Rheumatoid Arthritis
}

\author{
Mark R. Tanner, Michael W. Pennington, Brayden H. Chamberlain, Redwan Huq, \\ Elizabeth J. Gehrmann, Teresina Laragione, Pércio S. Gulko, and Christine Beeton
}

Department of Molecular Physiology and Biophysics (M.R.T., B.H.C., R.H., E.J.G., C.B.), Interdepartmental Graduate Program in Translational Biology and Molecular Medicine (M.R.T.), and Biology of Inflammation Center and Center for Drug Discovery (C.B.), Baylor College of Medicine, Houston, Texas; Peptides International Inc., Louisville, Kentucky (M.W.P.); and Division of Rheumatology, Department of Medicine, Icahn School of Medicine at Mount Sinai, New York, New York (T.L., P.S.G.)

Received September 11, 2017; accepted February 14, 2018

\begin{abstract}
Fibroblast-like synoviocytes (FLSs) are a key cell type involved in rheumatoid arthritis (RA) progression. We previously identified the KCa1.1 potassium channel (Maxi-K, BK, Slo 1, KCNMA1) as a regulator of FLSs and found that $\mathrm{KCa} 1.1$ inhibition reduces disease severity in RA animal models. However, systemic KCa1.1 block causes multiple side effects. In this study, we aimed to determine whether the KCa1.1 $\beta 1$-3-specific venom peptide blocker iberiotoxin (IbTX) reduces disease severity in animal models of RA without inducing major side effects. We used immunohistochemistry to identify lbTX-sensitive KCa1.1 subunits in joints of rats with a model of RA. Patch-clamp and functional assays were used to determine whether IbTX can regulate FLSs through targeting KCa1.1. We then tested the efficacy of IbTX in
\end{abstract}

ameliorating disease in two rat models of RA. Finally, we determined whether lbTX causes side effects including incontinence or tremors in rats, compared with those treated with the small-molecule KCa1.1 blocker paxilline. IbTX-sensitive subunits of KCa1.1 were expressed by FLSs in joints of rats with experimental arthritis. IbTX inhibited KCa1.1 channels expressed by FLSs from patients with RA and by FLSs from rat models of RA and reduced FLS invasiveness. IbTX significantly reduced disease severity in two rat models of RA. Unlike paxilline, IbTX did not induce tremors or incontinence in rats. Overall, IbTX inhibited KCa1.1 channels on FLSs and treated rat models of RA without inducing side effects associated with nonspecific KCa1.1 blockade and could become the basis for the development of a new treatment of RA.

\section{Introduction}

Rheumatoid arthritis (RA) is a chronic, systemic autoimmune disease affecting approximately 1.3 million people in the United States. It involves inflammation of the synovial joints (Helmick et al., 2008). Fibroblast-like synoviocytes (FLSs) are resident synovial joint cells that play a central role in the progression of RA. These cells develop a highly invasive phenotype and secrete proteases, growth factors, and proinflammatory cytokines and chemokines, leading to joint degradation (Bartok and Firestein, 2010; Bottini and Firestein, 2013). This invasive phenotype is a hallmark of FLSs in RA, as their level of ex vivo invasiveness is correlated with radiographic damage in patients with RA (Tolboom et al., 2005) and with histologic changes in rodents (Laragione et al., 2008).

This work was supported by the Arthritis Foundation [Grant 6483], the National Institutes of Health (NIH) National Institute of General Medical Sciences [Grant T32 GM088129], the NIH National Institute of Allergy and Infectious Diseases [Grant T32 AI053831], the NIH National Institute of Arthritis and Musculoskeletal and Skin Diseases [Grant F31 AR069960], the NIH National Heart, Lung, and Blood Institute [Grant T32 HL007676], the NIH National Cancer Institute [Grant P30 CA125123], and the NIH National Human Genome Research Institute [Grant UM1 HG006348].

https://doi.org/10.1124/jpet.117.245118.
Current therapeutics for RA are focused on suppressing the immune component of RA and no current therapeutics have been designed to specifically target FLSs (Kahlenberg and Fox, 2011).

FLSs from patients with RA (RA-FLSs) and from rats with the pristane-induced arthritis (PIA) model of RA express the KCa1.1 potassium channel (Maxi-K, BK, Slo 1, KCNMA1) at their plasma membrane (Hu et al., 2012; Tanner et al., 2015; Pethö et al., 2016; Beeton, 2017). Inhibiting the expression or function of this channel reduces FLS invasiveness, proliferation, and protease secretion, while modulating cellular adhesion and integrin regulation (Hu et al., 2012; Tanner et al., 2015, 2017a). Furthermore, blocking KCa1.1 with the smallmolecule inhibitor paxilline reduces disease severity in PIA and in the collagen-induced arthritis (CIA) rat models of RA (Tanner et al., 2015).

Although paxilline was efficacious at treating disease in these rat models of RA, its development as a potential therapeutic is limited by its side effects. Pore-forming $\alpha$ subunits of KCa1.1 are found in a variety of tissues, including the central nervous system (CNS), smooth muscle, testis, pancreas, and kidney, in addition to FLSs (Uebele et al., 2000; Poulsen et al., 2009). Paxilline blocks KCa1.1 through allosteric binding to the

ABBREVIATIONS: CIA, collagen-induced arthritis; CNS, central nervous system; FLS, fibroblast-like synoviocyte; IbTX, iberiotoxin; PIA, pristaneinduced arthritis; RA, rheumatoid arthritis; RMS, root mean square; RP-HPLC, reversed-phase high-performance liquid chromatography; TFA, trifluoroacetic acid. 
pore-forming $\alpha$ subunit (Sanchez and McManus, 1996) and its systemic administration leads to KCa1.1 block throughout the body, causing detrimental side effects that preclude its use in humans (Meredith et al., 2004; Imlach et al., 2008).

Although KCa1.1 $\alpha$ subunits have a wide tissue distribution, many KCa1.1 channels are physically associated with regulatory $\beta$ subunits that affect channel gating and activation and have restricted tissue distributions. Highly invasive, $\mathrm{CD} 44^{\text {high }}$, cadherin- $11^{\text {high }}$, and podoplanin ${ }^{\text {high }}$ RA-FLSs express KCa1.1 containing the $\beta 3$ subunit (Pethő et al., 2016). In contrast, $\mathrm{KCa} 1.1 \alpha$ in the CNS is associated with the $\beta 4$ subunit, while the $\beta 1$ subunit is expressed in smooth muscle cells. Because of the limited tissue distribution of KCa1.1 with the $\beta 3$ subunit (Poulsen et al., 2009; Mannowetz et al., 2013; Pethö et al., 2016), it may be possible to use a blocker that affects KCa1.1 $\alpha \beta 3$, but not KCa1.1 $\alpha \beta 4$, as a step toward a novel therapy of RA that would not induce side effects in the CNS and as a proof of concept that targeting specific subunits of KCa1.1 can be used for tissue-specific pharmacology.

Iberiotoxin (IbTX), a 37-amino-acid peptide from the venom of the Buthus tamulus scorpion (Galvez et al., 1990; Yu et al., 2016), is a selective KCa1.1 blocker that inhibits the channel when it contains the $\beta 1, \beta 2$, or $\beta 3$ subunits (Löhn et al., 2001; Lippiat et al., 2003; Li et al., 2007) while having no effect on KCa1.1 containing the $\beta 4$ subunit found in the CNS (Wang et al., 2014). IbTX may therefore inhibit FLSs through blocking KCa1.1 $\alpha \beta 3$, while leaving the CNS unaffected by sparing KCa1.1 $\alpha \beta 4$. If IbTX is efficacious in treating models of RA, it could serve as a scaffold for the development of KCa1.1 $\alpha \beta 3$ specific blockers for the treatment of RA.

Here, we tested the hypothesis that IbTX can be used to block KCa1.1 expressed by FLSs without inducing the side effects observed with global KCa1.1 blockade. We show that IbTX blocks KCa1.1 in RA-FLSs, PIA-FLSs, and CIA-FLSs and also reduces their ex vivo invasiveness. We also show that IbTX is effective at reducing disease severity in the PIA and CIA rat models of RA and that paxilline, but not IbTX, induces tremors and incontinence in rats. Taken together, our results indicate IbTX as a tool for targeting FLSs without affecting other cells expressing KCa1.1, thereby reducing side effects associated with global KCa1.1 blockade.

\section{Materials and Methods}

Animals. Female dark agouti rats (Envigo, East Millstone, NJ) and Lewis rats (Charles River Laboratories, Wilmington, MA) aged 8-11 weeks were housed in autoclaved cages in an Association for Assessment and Accreditation of Laboratory Animal Care-accredited facility and given food and water ad libitum. All experiments involving rats were approved by the Institutional Animal Care and Use Committee at Baylor College of Medicine.

Immunohistochemistry, Histology, and X-Rays. Hind paws from rats with either CIA or PIA were collected 14 or 21 days after disease onset, respectively, at which time the disease had been severe for a long duration and animals had noticeable significant pathologic hallmarks of disease. X-rays were taken with an In Vivo Xtreme Imaging System (Bruker, Billerica, MA). Paws were decalcified, embedded in paraffin, sectioned, and stained for either safranin O/fast green or hematoxylin/eosin or by immunohistochemistry. Analysis and scoring of safranin O/fast green and hematoxylin/eosin-stained samples was completed as described (Brenner et al., 2005) by an investigator blinded to the treatment groups. For immunohistochemistry, sections were deparaffinized and hydrated before antigen retrieval and blocking of nonspecific protein binding sites, peroxidase, and alkaline phosphatase. Sections were incubated in primary antibodies against cadherin-11 (MA1-06306; Invitrogen, Carlsbad, CA), podoplanin (ab10288; Abcam, Cambridge, UK), KCa1.1 $\alpha$ (NeuroMab 75-022 from Antibodies Inc., Davis, CA; or AB5228 from EMD Millipore, Burlington, MA), and KCa1.1 $\beta 3$ (PA5-32891; Invitrogen). For detection, we used ImmPRESS anti-mouse and anti-rabbit IgG conjugated to alkaline phosphatase and peroxidase, respectively, followed by ImmPACT DAB and Vector Red substrates (Vector Laboratories, Burlingame, CA). Images of the synovial joints were taken using an Olympus Q Color 5 camera on an Olympus BX41 microscope (Olympus, Tokyo, Japan) at $10 \times$ magnification and images of synovium were taken at $40 \times$ magnification.

Cells. FLSs from patients with RA, diagnosed according to criteria set forth by the American College of Rheumatology (Aletaha et al., 2010), were isolated as described (Laragione and Gulko, 2010). Rat FLSs were isolated as described (Laragione et al., 2008) from the hind paws of rats with either CIA or PIA that had signs of disease for 14 or 21 days, respectively, at which time disease had been severe for a long duration. All experiments were conducted with FLSs after passage 3.

Synthesis of IbTX. IbTX was synthesized on a Prelude Peptide Synthesizer (Protein Technologies, Tucson, AZ) using an Fmoc-tBu protecting strategy with all Cys residues protected as Cys(Trt). Synthesis was initiated on Fmoc-Gln(Trt)-Wang resin (Peptides International, Louisville, KY) using diisopropyl carbodiimide/Oxyma activation chemistry. After synthesis of the linear sequence, the final pyroglutamic acid was coupled manually also using diisopropyl carbodiimide/Oxyma. The peptide was cleaved from the resin using an acidolytic cleavage cocktail [trifluoroacetic acid (TFA)/anisole/ $\mathrm{H}_{2} \mathrm{O} /$ triisopropyl silane/thioanisole, 9:0.2:0.2:0.2:0.2 (v/v)] for 2 hours at room temperature. After cleavage from the resin and deprotection of the side chain residues, the crude peptide was filtered to remove the spent resin beads and precipitated into ice-cold diethyl ether and washed five times with the same solvent. The crude peptide was subsequently dissolved in $50 \%$ aqueous acetic acid and diluted into water to a concentration of $0.2 \mathrm{mg} / \mathrm{ml}$. The $\mathrm{pH}$ of this solution was adjusted to 7.8 with $\mathrm{NH}_{4} \mathrm{OH}$ and glutathione was added to facilitate disulfide formation $(0.1 \mathrm{mM}$ glutathione disulfide and glutathione). After 18-hour oxidation, the peptide was monitored by reversed-phase high-performance liquid chromatography (RP-HPLC) for formation of the folded peptide, which shifts to a more hydrophilic retention time. The $\mathrm{pH}$ of the folded peptide solution was subsequently lowered with TFA to 4.0 and pumped onto a $5-\times 60-\mathrm{cm}$ preparative RP-HPLC column (Luna C18-10 u, $100 \AA$ pore size; Phenomenex, Torrance, CA). The folded peptide was purified using a gradient of $5 \%-35 \%$ acetonitrile versus $0.05 \%$ TFA in $\mathrm{H}_{2} \mathrm{O}$ over 90 minutes. The peptide fractions with a purity greater than $95 \%$ by analytical RP-HPLC were pooled and lyophilized. The final peptide was characterized by amino acid analysis and electrospray ionization-mass spectrometry and coeluted with a reference sample of IbTX. Each batch was tested by electrophysiology for KCa1.1 block on human embryonic kidney 293 cells expressing KCa1.1 $\alpha$.

Patch-Clamp Electrophysiology. FLSs were lifted with trypsinEDTA, washed, and patch clamped using a Port-a-Patch automated system (Nanion Technologies, Munich Germany), as described (Hu et al., 2012; Pethő et al., 2016; Tajhya et al., 2016).

Measuring FLS Invasiveness. Invasiveness of FLSs was determined as described (Laragione et al., 2008; Hu et al., 2012).

Induction and Scoring of RA Models. PIA was induced in female dark agouti rats by a subcutaneous injection of $150 \mu$ l pristane (MP Biomedicals, Santa Ana, CA) at the base of the tail, as described (Beeton et al., 2006; Tarcha et al., 2012). CIA was induced in female Lewis rats (Griffiths et al., 1992) by a subcutaneous injection of $200 \mu \mathrm{l}$ of $2 \mathrm{mg} / \mathrm{ml}$ porcine type II collagen (Chondrex, Redmond, WA) in a 1:1 emulsion with incomplete Freund's adjuvant (Difco, Detroit, MI). Seven days later, rats were given a booster injection of $100 \mu \mathrm{l}$ of the same emulsion.

Disease onset was defined by at least one swollen or red joint. Clinical scores were determined daily for each rat as described (Tarcha 
et al., 2012). For each rat, five points were given for each red or swollen ankle or wrist and one point for each red or swollen toe joint, with a maximum possible score of 60 for each rat. Upon disease onset, rats were randomly assigned to receive either vehicle, IbTX subcutaneously, or paxilline intraperitoneally every other day. Randomization of rats to treatment groups was done by assigning every other rat that developed signs of disease on a given day to the same treatment group so that each group was composed of rats that developed signs of disease across the same timeframe and without regard to basal disease severity on the day each rat had noticeable paw inflammation. The number of rats per treatment group was determined using power analysis with a power of 0.8 and an $\alpha$ of 0.5 and based off of previous experiments testing KCa1.1 blockers in rat models of RA, as described (Tanner et al., 2015).

Tremor Measurements. Lewis rats were treated with either dimethylsulfoxide, $20 \mathrm{mg} / \mathrm{kg}$ paxilline, or $0.5 \mathrm{mg} / \mathrm{kg} \mathrm{IbTX}$ and placed in a suspended chamber 10 minutes, 1 hour, and 24 hours after treatment. An iPhone 6 (Apple, Cupertino, CA) was attached to the bottom, outer surface of the chamber and tremors were measured through the phone's built-in accelerometer with vibration measurement and recording completed with the VibSensor application (Now Instruments and Software Inc., Poway, CA) (Miyazaki et al., 2014) over the course of 10 minutes. The acceleration data were root mean square (RMS) transformed with smoothing across 0.1-second increments, as described (Feketa et al., 2013). To determine tremor frequency over 10 minutes, a threshold above background RMStransformed acceleration of $0.15 \mathrm{~m} / \mathrm{s}^{2}$ was set and a single tremor event was counted as when the RMS-transformed acceleration passed through this threshold. To determine tremor intensity, the integral of the RMS-transformed acceleration was taken for a 1-minute period in which the rat was not actively moving. Rats were monitored over the course of measurements and data were excluded when a rat was moving.

Incontinence Assays. Lewis rats were given either an intraperitoneal treatment of vehicle or $20 \mathrm{mg} / \mathrm{kg}$ paxilline (Fermentek, Jerusalem, Israel) or a subcutaneous treatment of $0.5 \mathrm{mg} / \mathrm{kg} \mathrm{IbTX}$ and then given an oral gavage of $1 \mathrm{ml}$ water. They were then placed individually in paper towel-lined cages. After 1 hour, the number of urine spots each rat produced was counted in the dark using a black light (Meredith et al., 2004).

Statistical Analysis. All data are presented as the mean \pm S.E.M. Differences between groups were determined using Mann-Whitney $U$ tests or matched-pairs $t$ tests. A $P$ value less than 0.05 was considered statistically significant.

\section{Results}

Detection KCa1.1 $\beta 3$ in CIA Joints. We previously found that FLSs from rats with PIA express KCa1.1 and that their invasion is modulated by channel activity and expression (Tanner et al., 2015). Because ion channel subunit expression can differ between species and animal models (Tanner and Beeton, 2018), we assayed the in vivo expression of FLS expression of KCa1.1 $\alpha$ and $\beta 3$ within the synovial paw joints of rats with CIA, using cadherin-11 and podoplanin as markers for FLSs. Cadherin- $11^{+}$and podoplanin ${ }^{+}$cells expressed KCa1.1 $\alpha$ and $\beta 3$ in CIA rat synovium (Fig. 1). These data indicate that FLSs express KCa1.1 with the $\beta 3$ subunit in vivo during CIA and therefore allow for the CIA rat model to be used to assess the effect of KCa1.1 blockers at targeting FLSs in vivo.

IbTX Blocks KCa1.1 Channels Expressed by FLSs and Reduces FLS Invasiveness. Since the ability of IbTX to block KCa1.1 channels depends on the regulatory $\beta$ subunits expressed (Wang et al., 2014) and channel sensitivity to modulators can vary between species (Tanner and Beeton,
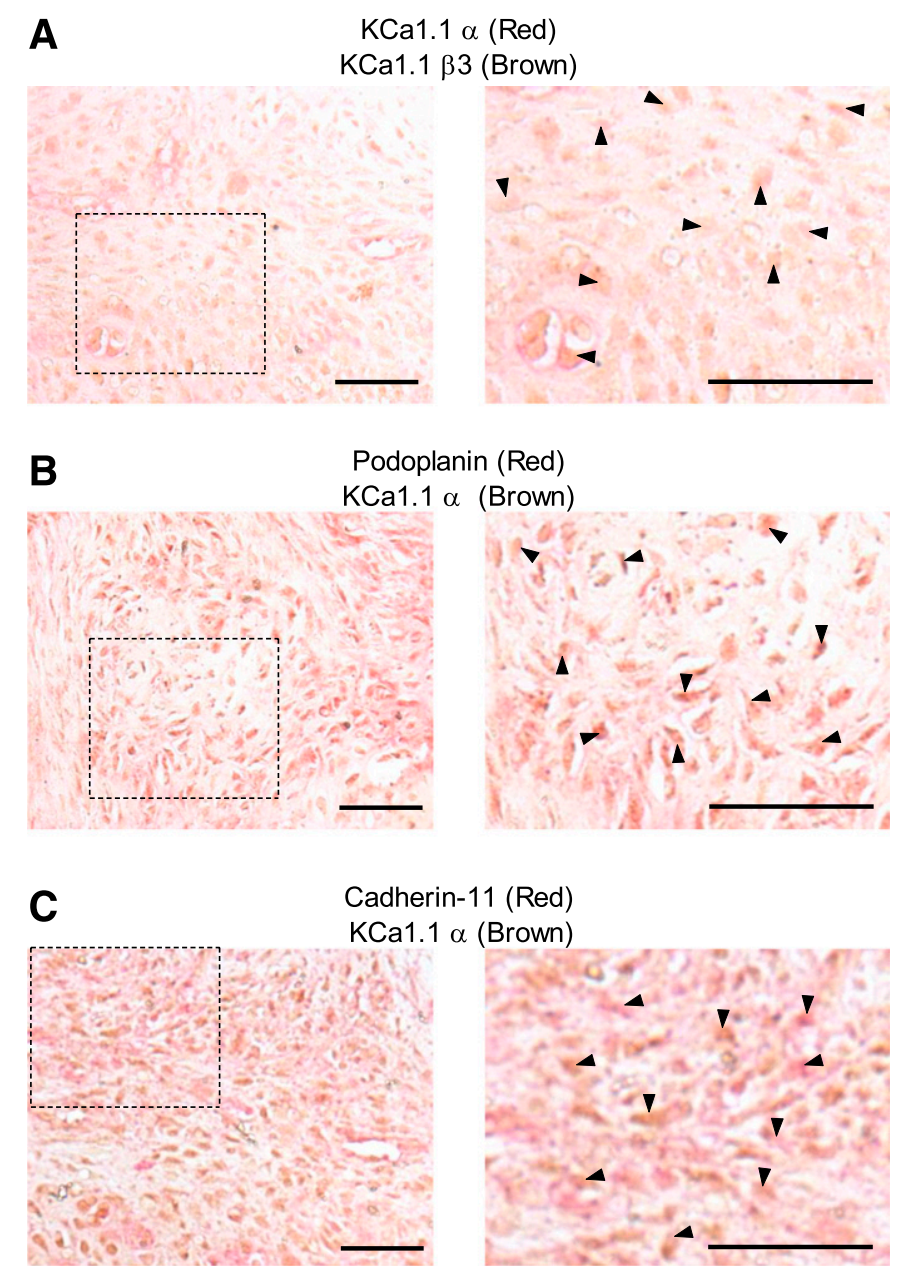

Fig. 1. KCa1.1 is expressed on CIA-FLSs in vivo. (A-C) Example images of synovial tissue from rats with CIA that had signs of disease for 14 days stained for expression of KCa1.1 $\alpha$ (red) and KCa1.1 $\beta 3$ (brown) (A), the FLS marker podoplanin (red) and KCa1.1 $\alpha$ (brown) (B), or the FLS marker cadherin-11 (red) and KCa1.1 $\alpha$ (brown) (C). Dashed boxes on the left demarcate the areas magnified to $80 \times$ shown on the right. Arrowheads point to double-stained cells. Scale bar, $50 \mu \mathrm{m}$. Original magnification, $40 \times$ (left) or $80 \times$ (right).

2018), we compared its effects on potassium channels from RA-, PIA-, and CIA-FLSs. IbTX blocked the potassium channels in all three cell types, with similar $\mathrm{IC}_{50}$ values in the 2- to 10-nM range previously described for IbTX on KCa1.1 lacking $\beta 4$ subunits (Tauc et al., 1993; Meera et al., 2000): $2.2 \pm 0.3 \mathrm{nM}$ for RA-FLSs, $2.6 \pm 0.7 \mathrm{nM}$ for PIA-FLSs, and $3.7 \pm 0.8 \mathrm{nM}$ for CIA-FLSs (Fig. $2 \mathrm{~A}$ ).

The small-molecule KCa1.1 blocker paxilline reduces the ex vivo invasiveness of RA- and PIA-FLSs (Hu et al., 2012; Tanner et al., 2015). Similar to paxilline, IbTX significantly reduced the number of RA-, PIA-, and CIA-FLSs that invaded through Matrigel-coated Transwell inserts (BD Biosciences, Franklin Lakes, NJ) by approximately 50\% (Fig. 2B).

IbTX Reduces Disease Severity in Two Rat Models of RA. Upon disease onset, rats with PIA were treated with vehicle or $0.05,0.5$, or $5 \mathrm{mg} / \mathrm{kg}$ IbTX subcutaneously every other day. These doses were chosen because venom peptides with a similar structure display efficacy in blocking their ion channel target at doses of $0.1-0.5 \mathrm{mg} / \mathrm{kg}$ in animal models of RA (Beeton et al., 2006; Tarcha et al., 2012; Tanner et al., 
A RA-FLS

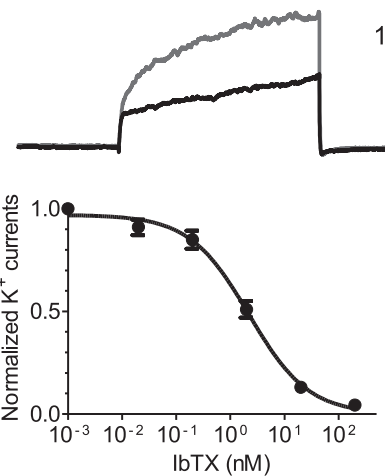

PIA-FLS

$1 \mathrm{nA} \bigsqcup_{100 \mathrm{~ms}}$

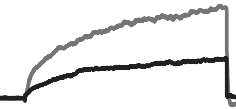

CIA-FLS

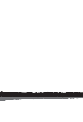

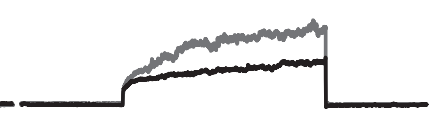

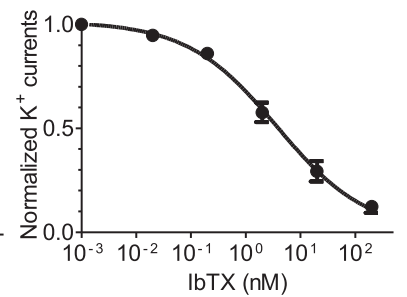

B

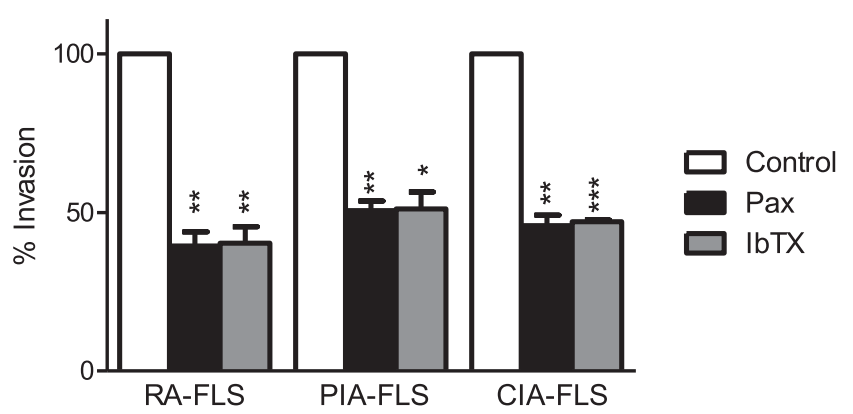

Fig. 2. IbTX inhibits KCa1.1 channels expressed on FLSs and reduces ex vivo FLS invasion. (A) Representative wholecell potassium currents (top) in RA-FLSs, PIA-FLSs, and CIA-FLSs before (gray) and after administration of $3 \mathrm{nM}$ IbTX (black) and dose-response inhibition of potassium currents by IbTX (bottom). (B) Invasiveness of FLSs from patients with RA and rats with either PIA or CIA through Matrigel-coated Transwell inserts in the presence of $10 \mu \mathrm{M}$ paxilline (Pax) or $10 \mu \mathrm{M}$ IbTX. Data are the mean \pm S.E.M. ( $n=3$ donors per group). $* P<0.05 ; * * P<$ $0.01 ; * * P<0.001$. 2017b). Rats treated with IbTX exhibited a greater than $50 \%$ reduction in arthritis severity clinical scores compared with those of vehicle-treated rats with PIA, with no differences observed between rats treated with the different doses of IbTX tested (Fig. 3A). In comparison, rats with PIA treated with $2 \mathrm{mg} / \mathrm{kg}$ paxilline exhibited only a slight reduction in disease severity, whereas those treated with $20 \mathrm{mg} / \mathrm{kg}$ paxilline exhibited a greater than $50 \%$ reduction in disease severity (Fig. 3B), similar to IbTX and in agreement with our previous findings (Tanner et al., 2015). Hematoxylin/eosin staining and safranin $\mathrm{O} /$ fast green staining of joint sections indicated that rats with PIA treated with $0.5 \mathrm{mg} / \mathrm{kg}$ IbTX had fewer immune infiltrates, pannus extensions, synovial hyperplasia, and cartilage erosions compared with vehicletreated animals (Fig. 3, C and D), whereas X-rays indicated that PIA rats treated with $0.5 \mathrm{mg} / \mathrm{kg}$ IbTX had significantly reduced bone damage compared with vehicle-treated rats (Fig. 3E). FLSs isolated from $0.5 \mathrm{mg} / \mathrm{kg}$ IbTX-treated PIA rats and cultured for at least three passages were significantly less invasive compared with FLSs isolated from vehicle-treated PIA rats and cultured for the same duration (Fig. 3F). Their invasiveness was comparable to that of FLSs from healthy rats.

To determine the efficacy of IbTX in rats that had already developed severe paw inflammation, mimicking the RA patient with established disease, rats with PIA were treated with $0.5 \mathrm{mg} / \mathrm{kg}$ IbTX or vehicle 7 days after they first exhibited signs of disease, at which point paw inflammation was already severe. PIA rats treated with IbTX every other day showed a significant decrease in paw inflammation after a 3- to 4-day lag time, whereas inflammation in vehicle-treated rats remained severe (Fig. 4A). To determine how long IbTX remains effective after cessation of treatment, rats with PIA were treated with $0.5 \mathrm{mg} / \mathrm{kg}$ IbTX for only the first 7 days after disease onset. IbTX-treated rats had significantly decreased paw inflammation on days 6 and 7 after the start of treatment; however, disease severity returned to levels comparable to vehicle after stopping treatment within 2 to 3 days (Fig. 4B).

To further verify the efficacy of IbTX in vivo, we tested it in the CIA model of RA. Rats with CIA were treated with either vehicle or $0.5 \mathrm{mg} / \mathrm{kg}$ IbTX every other day starting at disease onset. Similar to results in PIA, rats treated with IbTX had significantly less joint swelling compared with vehicle-treated rats (Fig. 5A). CIA-FLSs isolated from IbTX-treated rats and cultured for at least three passages were less invasive than CIA-FLSs isolated from vehicle-treated rats and cultured for the same duration (Fig. 5B). In addition, X-rays and histology of paws indicated less bone damage, as well as fewer immune infiltrates, pannus extensions, synovial hyperplasia, and cartilage erosions, in IbTX-treated animals compared with those treated with vehicle (Fig. 5, C-E).

Paxilline, But Not IbTX, Induces Tremors in Rats. KCa1.1 channels composed of the $\alpha$ and $\beta 4$ subunits are found in the CNS and loss of neuronal KCa1.1 expression or function causes tremors (Imlach et al., 2008; Wang et al., 2014). We therefore tested whether paxilline and IbTX induce tremors in rats. We chose doses of the blockers, $20 \mathrm{mg} / \mathrm{kg}$ paxilline and $0.5 \mathrm{mg} / \mathrm{kg}$ IbTX, which showed similar efficacy in the PIA and CIA models of RA in terms of clinical signs, joint inflammation, and cartilage degradation (Figs. 3 and 5) (Tanner et al., 2015). Vehicle-treated rats exhibited no tremors (Fig. 6A). Paxilline-treated rats exhibited abrupt events of increased acceleration (Fig. 6B), indicating the presence of tremor events. IbTX-treated rats exhibited no tremors and displayed acceleration measurements comparable to that of vehicletreated rats (Fig. 6C). By setting a threshold of $0.15 \mathrm{~m} / \mathrm{s}^{2}$ above the RMS-transformed data (Fig. 6, D-F) and counting the number of events the RMS-transformed acceleration passed 
A

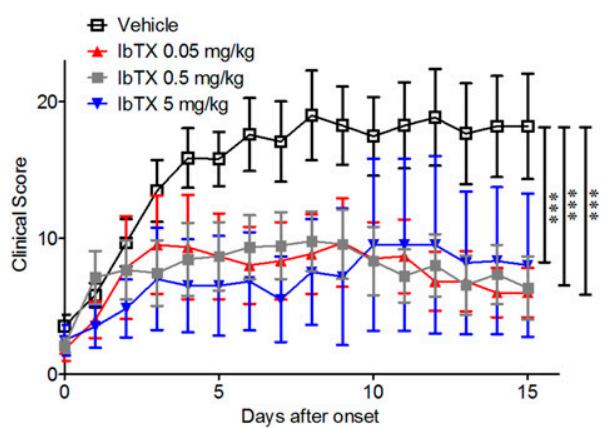

C

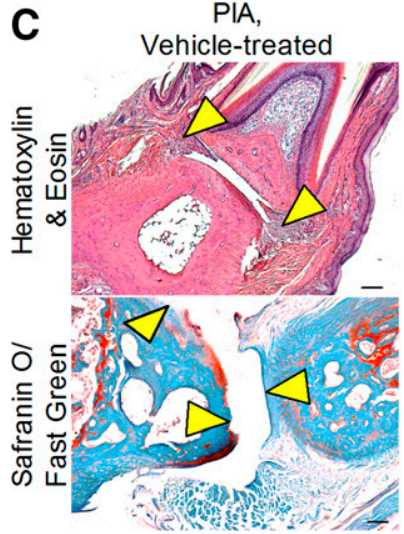

E
B

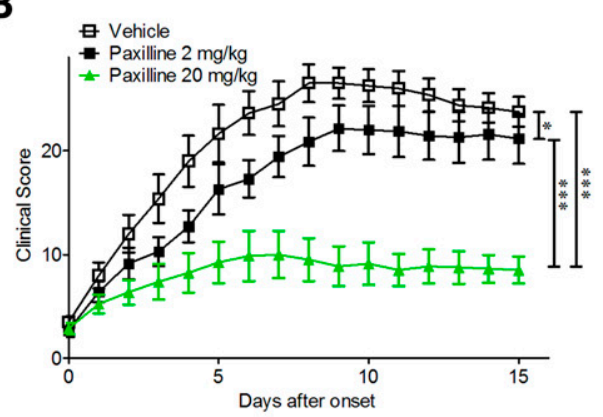

D
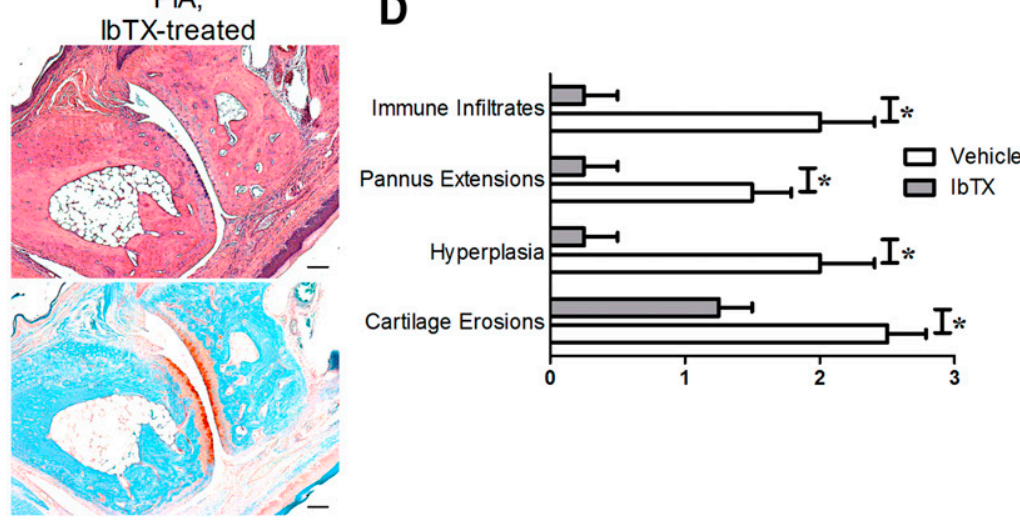

$\mathbf{F}$

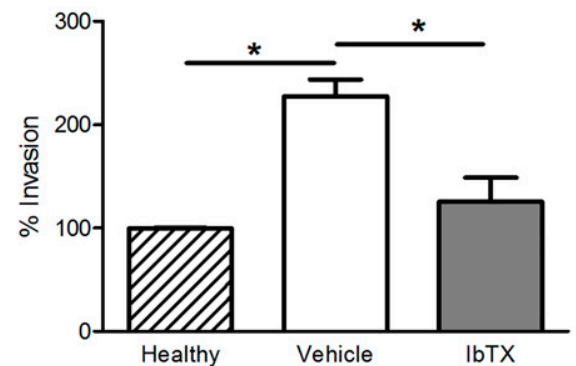

PIA,

Vehicle-treated

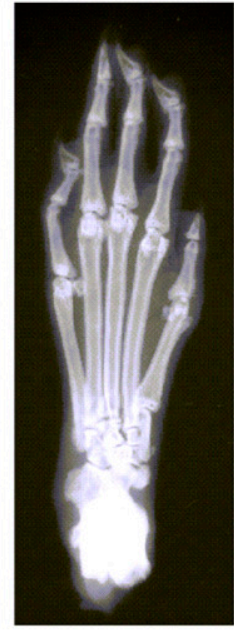

PIA,

IbTX-treated

Fig. 3. IbTX reduces signs of disease in PIA. (A) Clinical scores of paw inflammation from rats with PIA treated with vehicle (white) or $0.05 \mathrm{mg} / \mathrm{kg}$ (red), $0.5 \mathrm{mg} / \mathrm{kg}$ (gray), or $5 \mathrm{mg} / \mathrm{kg} \mathrm{IbTX} \mathrm{(blue)} \mathrm{subcutaneously} \mathrm{every} \mathrm{other} \mathrm{day} \mathrm{starting} \mathrm{at} \mathrm{disease} \mathrm{onset.} \mathrm{Data} \mathrm{are} \mathrm{the} \mathrm{mean} \pm$ S.E.M. $(n=6$ rats in the $0.05 \mathrm{mg} / \mathrm{kg}$ IbTX and $5 \mathrm{mg} / \mathrm{kg}$ IbTX groups, $n=9$ rats in the $0.5 \mathrm{mg} / \mathrm{kg}$ IbTX group, and $n=15 \mathrm{rats}$ in the vehicle-treatment group). (B) Clinical scores of paw inflammation from rats with PIA treated with vehicle (white), $2 \mathrm{mg} / \mathrm{kg}$ paxilline (black), or $20 \mathrm{mg} / \mathrm{kg}$ paxilline (green) intraperitoneally every other day starting at disease onset ( $n=7$ to 8 rats per group). (C) Hematoxylin/eosin staining (top) and safranin O/fast green staining (bottom) of tissue sections of paws from rats with PIA treated with vehicle (left) or IbTX (right). Arrowheads indicate areas of hyperplasia (hematoxylin/eosin) and cartilage erosions (safranin O/fast green). (D) Histology scoring of paw joints of rats with PIA treated with vehicle (white) or $0.5 \mathrm{mg} / \mathrm{kg}$ IbTX (gray). Data are the mean \pm S.E.M. ( $n=4$ paws per group). (E) Example X-rays of hind paws from vehicle-treated (left) and IbTX-treated (right) rats. (F) Ex vivo invasion of FLSs isolated from three healthy dark agouti rats, three rats with PIA treated with vehicle, and three rats with PIA treated with $0.5 \mathrm{mg} / \mathrm{kg}$ IbTX through Matrigel-coated Transwell inserts. Data are the mean \pm S.E.M. $\left(n=3\right.$ per group). ${ }^{*} P<0.05 ; * * * P<0.001$. Scale bar, $100 \mu \mathrm{m}$ in $(C)$.

above this threshold, we determined that paxilline-treated rats had approximately $14 \pm 4$ tremor events during the recordings 10 minutes after treatment (Fig. 6G). Tremor frequency decreased over time. In sharp contrast, vehicleand IbTX-treated rats exhibited no tremors (Fig. 6G). Quantifying tremors through the integral of the RMS-transformed acceleration determined that paxilline-treated rats had highintensity tremors soon after treatment, which decreased over time, while vehicle- and IbTX-treated rats did not exhibit such changes in acceleration intensity (Fig. $6 \mathrm{H}$ ). Taken together, these data indicate that paxilline, but not IbTX, induces tremors in rats. 

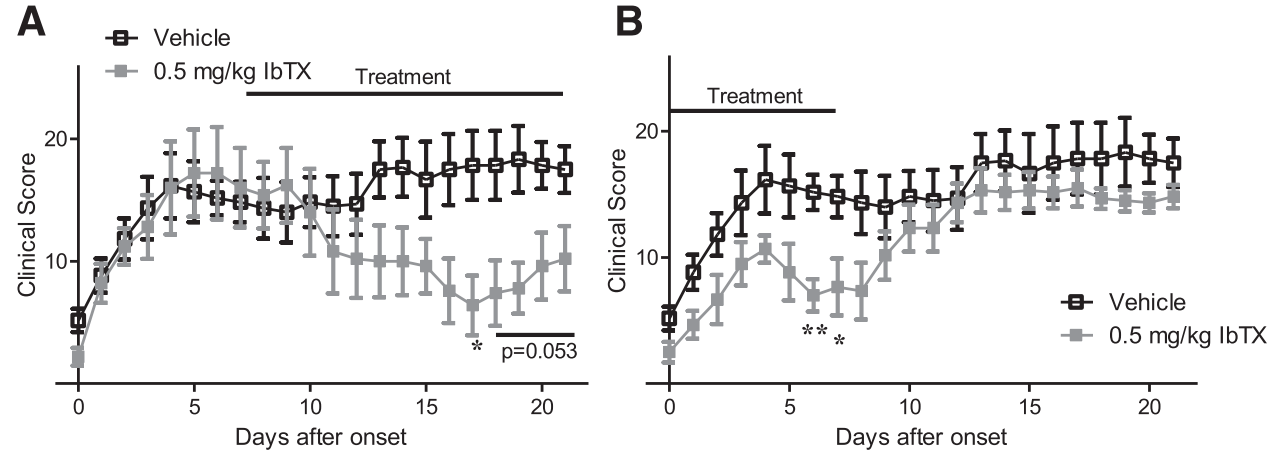

Fig. 4. (A) Clinical scores of paw inflammation from rats with PIA treated with vehicle (black) or with $0.5 \mathrm{mg} / \mathrm{kg}$ IbTX (gray) every other day starting 7 days after disease onset. Data are the mean \pm S.E.M. ( $n=5$ to 6 rats per group). (B) Clinical scores of rats with PIA treated with vehicle (black) or with IbTX (gray) every other day for the first 7 days after disease onset. Data are the mean \pm S.E.M. ( $n=6$ rats per group). $* P<0.05 ; * * P<0.01$.
Paxilline, But Not IbTX, Induces Incontinence in Rats. Considering that IbTX and paxilline both block KCa1.1 channels associated with the $\beta 1$ subunit that is found in smooth muscle, including that of the urinary bladder (Poulsen et al., 2009), we tested whether they cause incontinence in rats. Healthy Lewis rats were treated with either vehicle, paxilline, or IbTX. An hour later, paxilline-treated rats exhibited a large number of urine spots, indicating a lack of bladder control. Surprisingly, IbTX-treated animals exhibited a similar small number of urine spots as vehicle-treated rats (Fig. 7). This indicates that IbTX does not induce incontinence, whereas paxilline does.

\section{Discussion}

In this study, we validated the peptide KCa1.1 blocker IbTX for efficacy in reducing the ex vivo invasiveness of FLSs from patients with RA and from multiple rat models of RA. We also show that IbTX reduces disease severity in both the PIA and CIA rat models of RA, while not inducing tremors or incontinence. Together, these data suggest IbTX as a potential novel therapeutic for RA by inhibiting FLSs and without inducing side effects seen with broad KCa1.1 blockade.

No animal model of RA fully recapitulates all aspects of the human disease; we therefore assayed IbTX for efficacy in both PIA and CIA, two well established models of RA in rats. Although IbTX significantly reduced disease severity in both models to a similar degree as paxilline (Tanner et al., 2015), IbTX-treated animals still exhibited a significant amount of paw inflammation. The inability of KCa1.1 blockers to eliminate inflammation may be due to other cell types involved in RA such as $\mathrm{T}$ lymphocytes, chondrocytes, and osteoclasts, which may still have pathogenic phenotypes after IbTX treatment (Lundy et al., 2007; Otero and Goldring, 2007; Schett, 2007). IbTX likely does not have an effect on $\mathrm{T}$ lymphocytes in RA because these cells express different potassium channels from KCa1.1, which are insensitive to IbTX and paxilline (Beeton and Chandy, 2005; Beeton et al., 2011). Osteoclasts and chondrocytes express several potassium channels, including KCa1.1 (Arkett et al., 1994; Weidema et al., 2000; Mobasheri et al., 2012). The regulatory $\beta$ subunits of KCa1.1 expressed by these cells and whether IbTX has an effect on their destructive functions during RA have yet to be investigated. It is possible that a combined therapy consisting of inhibitors specific for each of these cell types will be needed to further reduce disease severity in models of RA. Alternatively, it may be possible for a combined treatment of IbTX with a currently available RA therapy, such as a tumor necrosis factor- $\alpha$ antagonist, to have an additive effect at treating RA by using IbTX to target FLSs and a currently available therapy to reduce inflammation.

We examined the effect of KCa1.1 blockers on the in vitro invasion of FLSs that were passaged multiple times. From this, we infer that KCa1.1 blockers reduce disease severity in rat models of RA at least in part from reducing FLS invasion in vivo. Passaging these cells is a standard practice to isolate FLSs and is necessary to obtain a population solely composed of FLSs without any contaminating macrophage-like synoviocytes, synovial-infiltrating immune cells, or endothelial cells that either die or are washed away through multiple in vitro passages (Rosengren et al., 2007; Bartok and Firestein, 2010). Passaged RA-FLSs and PIA-FLSs maintain a high level of invasiveness and secrete proinflammatory cytokines and proteases after passaging, indicating that passaged FLSs maintain a pathogenic phenotype in vitro (Laragione et al., 2008; Bartok and Firestein, 2010; Tanner et al., 2015). The in vitro invasion of passaged FLSs is also directly correlated with radiographic damage in patients with RA, as well as in PIA (Tolboom et al., 2005; Laragione et al., 2008). Furthermore, our data indicate that FLSs in the synovium in situ of rats with CIA express KCa1.1 with the $\beta 3$ subunit, supporting the notion that FLSs maintain similar potassium channel phenotypes in vivo and in vitro. Therefore, it is likely that the potassium channel phenotype of FLSs and effects of KCa1.1 blockers on FLS phenotypes are similar in vitro and in vivo.

PIA rats treated with IbTX for only the first week after disease onset exhibited a rapid increase in joint swelling once treatment ceased, indicating that IbTX was only efficacious with continuous administration. This may be due to proteolytic degradation of the peptide in vivo. Alternatively, as IbTX has a molecular weight of only $4.231 \mathrm{kDa}$ (below the cutoff of $30-50 \mathrm{kDa}$ for glomerular filtration), it could be rapidly eliminated by renal clearance. The addition of polyethylene glycol moieties is a widely used method to increase the length of efficacy of therapeutics by reducing renal clearance and decreasing the rate of proteolytic degradation (Harris and Chess, 2003; Turecek et al., 2016). Creating a PEGylated IbTX analog could therefore be used to increase its length of efficacy. Indeed, such an approach has successfully been used to improve the duration of efficacy of other venomderived peptide potassium channel blockers (Tanner et al., 2017b).

In this study, we used a smartphone's accelerometer to measure tremors in rats. Other techniques used to measure tremors in laboratory settings involve using electrodes implanted 

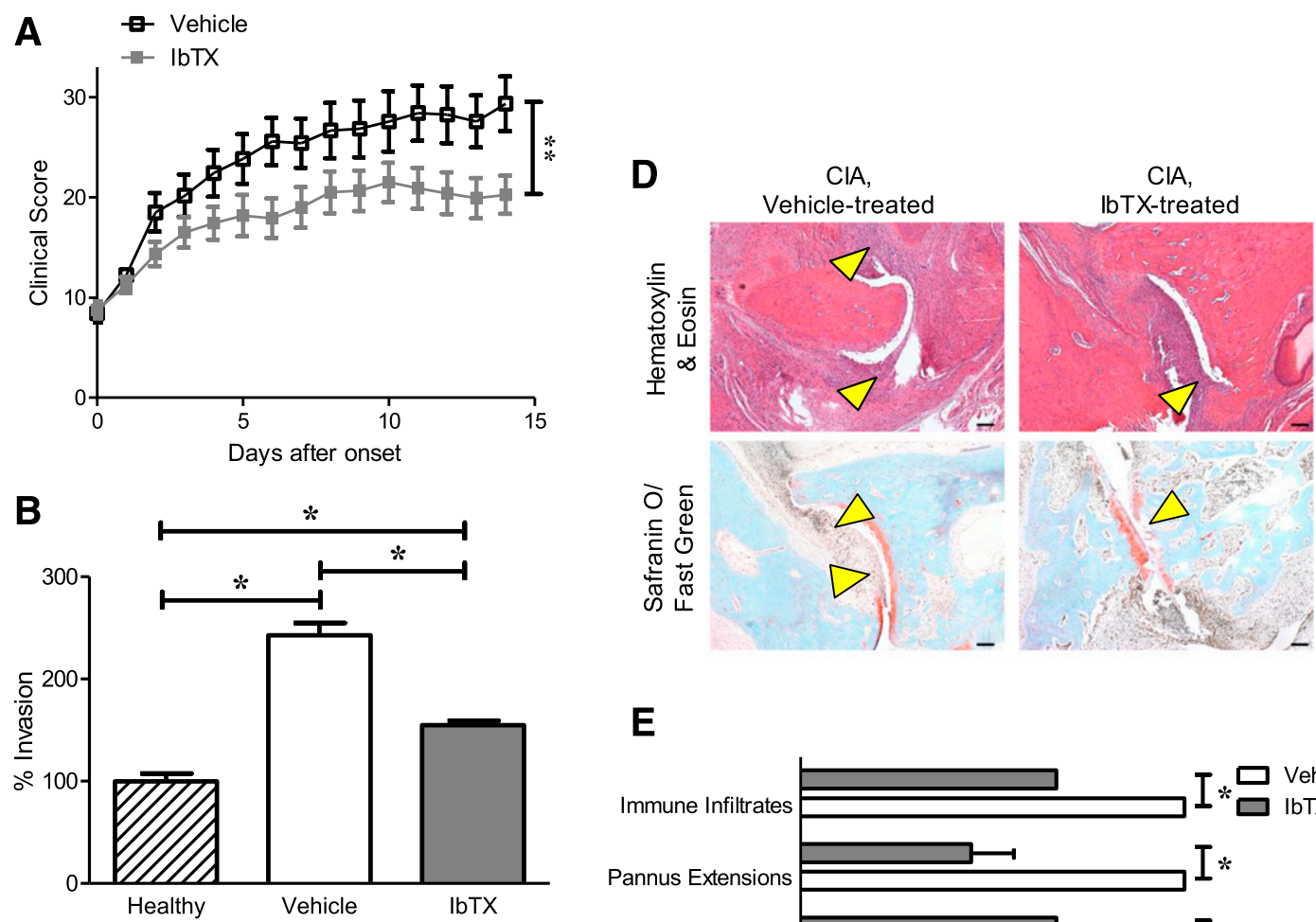

E

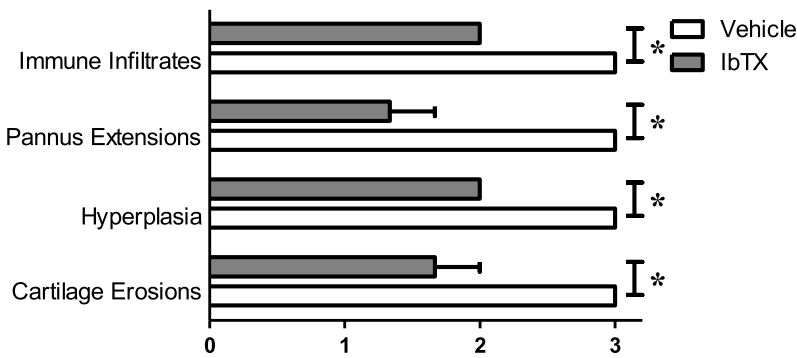

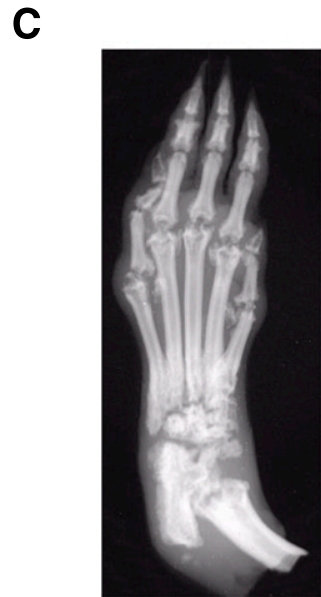

CIA,

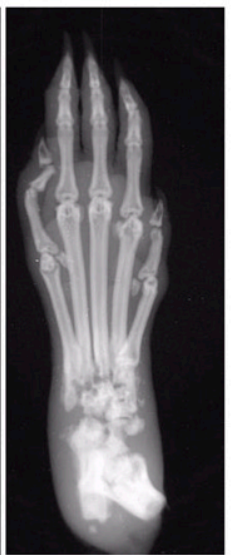

CIA,

Vehicle-treated IbTX-treated

Fig. 5. IbTX reduces disease severity in the CIA rat model of RA. (A) Clinical scores of paw inflammation of rats with CIA treated with vehicle (black) or $0.5 \mathrm{mg} / \mathrm{kg}$ IbTX (gray) every other day starting at disease onset. Data are the mean \pm S.E.M. ( $n=12$ rats per group). (B) Ex vivo invasiveness of FLSs from three healthy Lewis rats, three rats with CIA treated with vehicle, and three rats with CIA treated with $0.5 \mathrm{mg} / \mathrm{kg}$ IbTX through Matrigel-coated Transwell inserts. Data are the mean \pm S.E.M. $(n=3$ per group). (C) Example X-ray images of paws from rats with CIA treated every other day with vehicle (left) or IbTX (right) for 14 days after disease onset. (D) Hematoxylin/eosin staining (top) and safranin O/fast green staining (bottom) of tissue sections of paws from rats with CIA treated with vehicle (left) or IbTX (right). Arrowheads indicate areas of hyperplasia (hematoxylin/eosin) and cartilage erosions (safranin O/fast green). (E) Histology scoring of paw joints of rats with CIA treated with vehicle (white) or IbTX (gray). Data are the mean \pm S.E.M. $\left(n=3\right.$ paws per group). ${ }^{*} P<0.05 ; * * P<0.01$. Scale bar, $100 \mu \mathrm{m}$ in (D).

into the back muscles of rats and measuring contractions over time (Feketa et al., 2013). Such techniques involve the use of specialized equipment and software, as well as survival surgeries that are stressful for the animals. Our technique using a smartphone's accelerometer is noninvasive and minimally stressful for the rats, as it requires no surgery, and is more cost-effective compared with surgical interventions. In addition, this technique was effective at quantifying the tremors observed in paxilline-treated rats and at differentiating the presence and absence of tremors, making it a valuable technique to quickly screen whether potential drugs are tremorigenic in vivo. Similar accelerometer-based technologies have also been used to observe tremors in rats and mice (Shih and Young, 2007; Miyazaki et al., 2014) and smartphone-based accelerometry applications have even been used to monitor tremors in patients with Parkinson's disease (Joundi et al., 2011; Kostikis et al., 2015).

Paxilline given intraperitoneally at $20 \mathrm{mg} / \mathrm{kg}$ every other day reduces signs of disease in rat models of $\mathrm{RA}$, whereas $2 \mathrm{mg} / \mathrm{kg}$ given at the same frequency has only minimal 


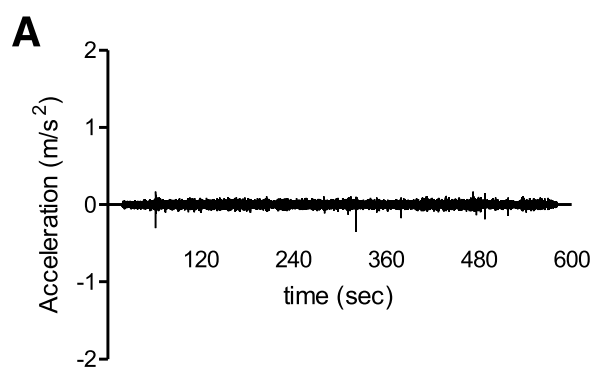

B

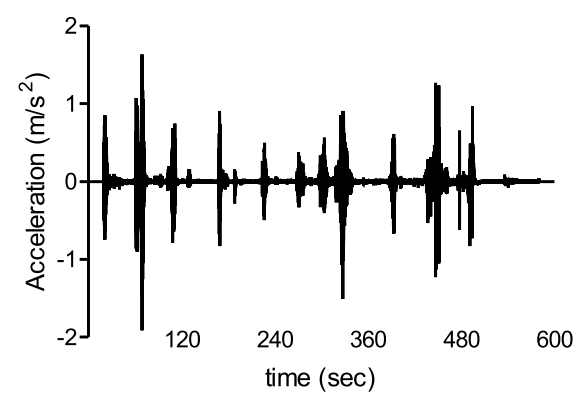

C

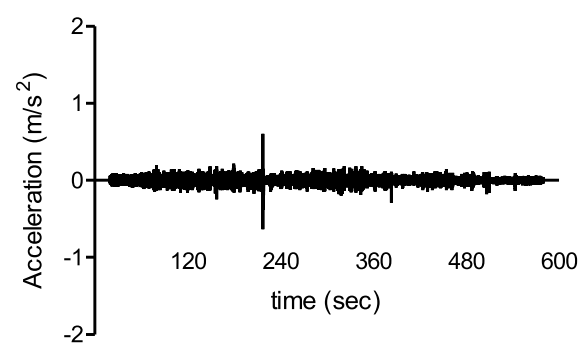

G



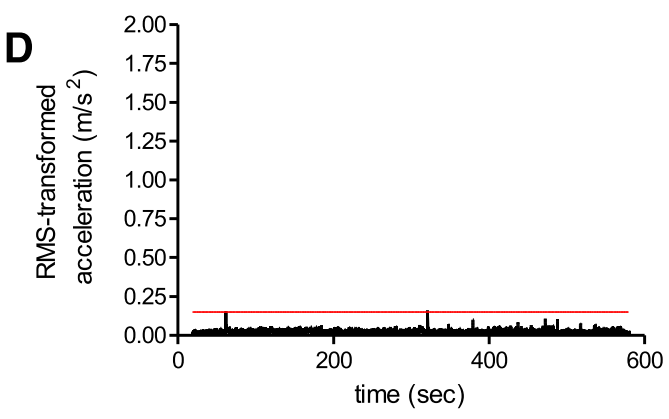

E

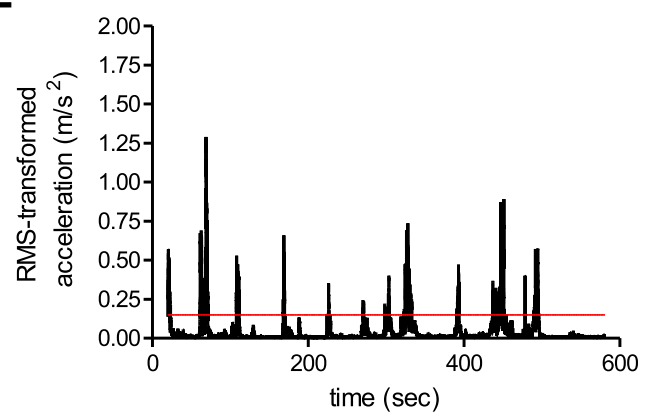

$\mathbf{F}$

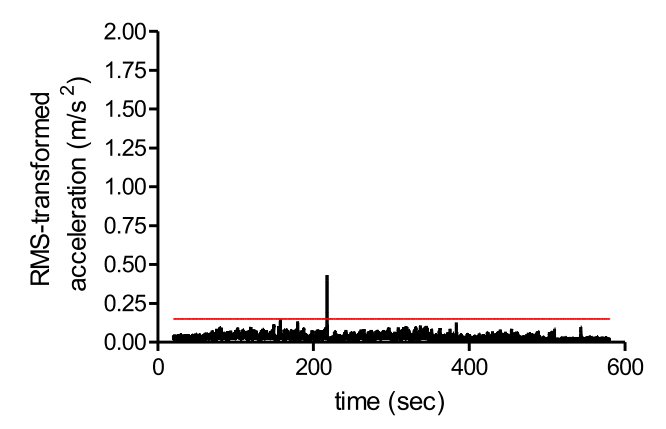

H

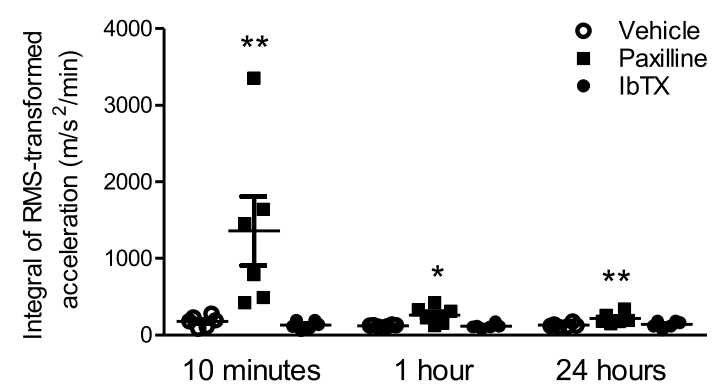

Fig. 6. Paxilline, but not IbTX, causes tremors in rats. (A-C) Example acceleration recordings of rats treated with vehicle (A), $20 \mathrm{mg} / \mathrm{kg}$ paxilline (B), or $0.5 \mathrm{mg} / \mathrm{kg} \mathrm{IbTX}(\mathrm{C})$. (D-F) RMS-transformed accelerations from the raw data presented in (A) to (C). Red lines indicate the threshold above background acceleration. (G) Numbers of tremor events above the threshold of the RMS-transformed acceleration of rats treated with vehicle, paxilline, or IbTX 10 minutes, 1 hour, and 24 hours after treatment. Data are the mean \pm S.E.M. ( $n=6$ rats per group). (H) Tremor intensity of rats treated with vehicle, paxilline, or IbTX 10 minutes, 1 hour, and 24 hours after treatment. Data are the mean \pm S.E.M. $\left(n=6\right.$ rats per group). ${ }^{*} P<0.05 ; * * P<0.01$ compared with the vehicle-treated group.

improvement in clinical signs of disease (Tanner et al., 2015). IbTX at doses of $0.05-5 \mathrm{mg} / \mathrm{kg}$ subcutaneously every other day gave a similar efficacy as paxilline in treating CIA and PIA. This discrepancy in dosing efficacy was due to venomderived potassium channel blocking peptides creating a depot under the skin when given subcutaneously and entering the circulation over hours (Tarcha et al., 2012). This allows for small doses to provide sustained benefit, as opposed to giving a bolus of paxilline intraperitoneally with a half-life of less than 1 hour (Tanner et al., 2015).

Paxilline is a well known tremorgenic compound, as it is a lipophilic small molecule that can cross membranes to enter the CNS and other tissues and blocks KCa1.1 channels regardless of $\beta$ subunit expression (Imlach et al., 2008). For these reasons, we also expected that paxilline would induce incontinence (Meredith et al., 2004). The lack of tremor 


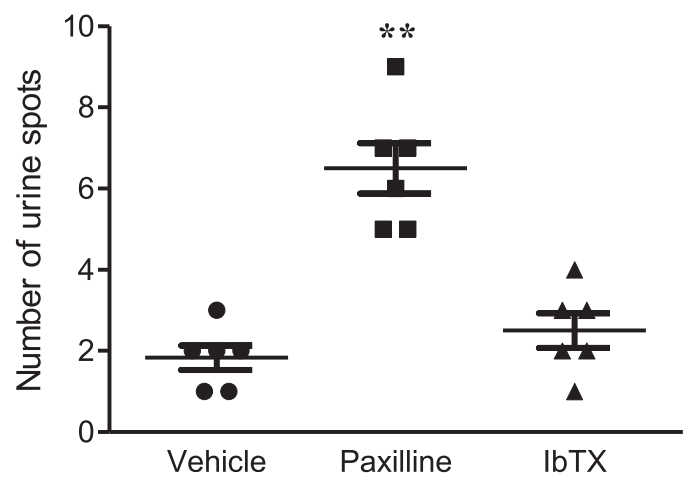

Fig. 7. Paxilline, but not IbTX, induces incontinence in rats. The number of urine spots counted from healthy Lewis rats treated with either vehicle, $20 \mathrm{mg} / \mathrm{kg}$ paxilline, or $0.5 \mathrm{mg} / \mathrm{kg}$ IbTX and then given an oral gavage of water and placed individually in a paper towel-lined cage for 1 hour. Data are the mean \pm S.E.M. ( $n=6$ rats per group). ${ }^{*} P<0.01$ compared with the vehicle-treated group.

induction by IbTX was anticipated, as this peptide blocks KCa1.1 only in the absence of $\beta 4$ subunits and these subunits are widely expressed in the CNS. The lack of incontinence in rats treated with IbTX was surprising, because this is induced by the targeting of KCa1.1 expressed by smooth muscle cells in the urinary bladder. These cells express the $\beta 1$ subunit of KCa1.1 and IbTX blocks KCa1.1 containing this subunit. However, smooth muscle cells are separated from the circulation by endothelial cells and from the urine by bladder epithelial cells. IbTX is likely unable to cross these barriers and therefore unable to reach the smooth muscle cell KCa1.1 channels.

Our studies of potential side effects associated with blocking KCa1.1 focused on tremors and incontinence. However, due to the tissue distribution of this channel, there may be other side effects from blocking KCa1.1. These include hypertension, as KCa1.1 associated with the $\beta 1$ subunit is found in the smooth muscle of blood vessels in rats (Brenner et al., 2000; Poulsen et al., 2009). Given our results indicating a lack of effect of IbTX on the urinary bladder, it is likely that IbTX also does not affect vascular smooth muscle, which is separated from the circulation by a layer of endothelium that IbTX may not be able to cross. Male fertility may also be affected, as KCa1.1 associated with the $\beta 3$ subunit is found in the rat testis and human spermatozoa (Poulsen et al., 2009; Mannowetz et al., 2013). However, IbTX may not be able to cross through the blood-testis barrier. Furthermore, KCa1.1 associated with the $\beta 2$ subunit is found in the human pancreas and kidney and blocking this channel may have detrimental effects on these tissues as well (Uebele et al., 2000). To more completely validate IbTX as a therapeutic for use in humans, these side effects associated with KCa1.1 block in these tissues will need to be examined. It is indeed possible that IbTX, as well as paxilline, will induce side effects from affecting these tissues, as IbTX can block KCa1.1 associated with subunits $\beta 1-\beta 3$ or with no $\beta$ subunits. If this is the case, it may be necessary to identify any unique splice variants of KCa1.1 pore-forming $\alpha$ subunits expressed by FLSs and generate a KCa1.1 blocker specific for this unique aspect, along with the $\beta 3$ subunit, of the channel to create a more viable therapeutic for RA. Regardless, our findings that IbTX does not induce tremors or incontinence show that selectively inhibiting KCa1.1 in a $\beta$ subunit-specific manner can be a viable means of treating diseases mediated by this potassium channel without inhibiting all variants of this channel.

Overall, this study indicates the KCa1.1 potassium channel blocker IbTX as an inhibitor of the invasive phenotype of FLSs and that IbTX reduces disease severity in two rat models of RA. We have also shown that KCa1.1 block with IbTX eliminates two major side effects observed during systemic KCa1.1 blockade with small molecules and in mice lacking KCa1.1 $\alpha$ expression (Meredith et al., 2004; Imlach et al., 2008). Taken together, these data indicate IbTX as either a potential novel therapeutic for the treatment of RA by targeting KCa1.1 channels expressed on FLSs or as a scaffold for the future generation of RA-FLS-selective KCa1.1 blockers.

\section{Acknowledgments}

We thank Dr. Sean Marrelli (Department of Anesthesiology, Baylor College of Medicine, Houston, TX) for assisting with the calculations and data analysis of tremor measurements. We also acknowledge excellent service from the Mouse Metabolism and Phenotyping and Pathology and Histology Cores at Baylor College of Medicine.

\section{Authorship Contributions}

Participated in research design: Tanner, Beeton.

Conducted experiments: Tanner, Chamberlain, Huq, Gehrmann, Beeton.

Contributed new reagents or analytic tools: Pennington, Laragione, Gulko.

Performed data analysis: Tanner, Beeton.

Wrote or contributed to the writing of the manuscript: Tanner, Pennington, Chamberlain, Huq, Gehrmann, Laragione, Gulko, Beeton.

\section{References}

Aletaha D, Neogi T, Silman AJ, Funovits J, Felson DT, Bingham Co, 3rd, Birnbaum NS, Burmester GR, Bykerk VP, Cohen MD, et al. (2010) 2010 rheumatoid arthritis classification criteria: an American College of Rheumatology/European League Against Rheumatism collaborative initiative. Arthritis Rheum 62:2569-2581.

Arkett SA, Dixon J, Yang JN, Sakai DD, Minkin C, and Sims SM (1994) Mammalian osteoclasts express a transient potassium channel with properties of Kv1.3. Receptors Channels 2:281-293.

Bartok B and Firestein GS (2010) Fibroblast-like synoviocytes: key effector cells in rheumatoid arthritis. Immunol Rev 233:233-255.

Beeton C (2017) KCa1.1 channels as therapeutic targets for rheumatoid arthritis. Expert Opin Ther Targets 21:1077-1081.

Beeton C and Chandy KG (2005) Potassium channels, memory T cells, and multiple sclerosis. Neuroscientist 11:550-562.

Beeton C, Pennington MW, and Norton RS (2011) Analogs of the sea anemone potassium channel blocker ShK for the treatment of autoimmune diseases. Inflamm Allergy Drug Targets 10:313-321.

Beeton C, Wulff H, Standifer NE, Azam P, Mullen KM, Pennington MW, KolskiAndreaco A, Wei E, Grino A, Counts DR, et al. (2006) Kv1.3 channels are a therapeutic target for T cell-mediated autoimmune diseases. Proc Natl Acad Sci USA 103:17414-17419.

Bottini N and Firestein GS (2013) Duality of fibroblast-like synoviocytes in RA: passive responders and imprinted aggressors. Nat Rev Rheumatol 9:24-33.

Brenner M, Meng HC, Yarlett NC, Griffiths MM, Remmers EF, Wilder RL, and Gulko PS (2005) The non-major histocompatibility complex quantitative trait locus Cia10 contains a major arthritis gene and regulates disease severity, pannus formation, and joint damage. Arthritis Rheum 52:322-332.

Brenner R, Peréz GJ, Bonev AD, Eckman DM, Kosek JC, Wiler SW, Patterson AJ, Nelson MT, and Aldrich RW (2000) Vasoregulation by the beta1 subunit of the calcium-activated potassium channel. Nature 407:870-876.

Feketa VV, Balasubramanian A, Flores CM, Player MR, and Marrelli SP (2013) Shivering and tachycardic responses to external cooling in mice are substantially suppressed by TRPV1 activation but not by TRPM8 inhibition. Am J Physiol Regul Integr Comp Physiol 305:R1040-R1050.

Galvez A, Gimenez-Gallego G, Reuben JP, Roy-Contancin L, Feigenbaum P, Kaczorowski GJ, and Garcia ML (1990) Purification and characterization of a unique, potent, peptidyl probe for the high conductance calcium-activated potassium channel from venom of the scorpion Buthus tamulus. J Biol Chem 265: $11083-11090$.

Griffiths MM, Cremer MA, Harper DS, McCall S, and Cannon GW (1992) Immunogenetics of collagen-induced arthritis in rats. Both MHC and non-MHC gene products determine the epitope specificity of immune response to bovine and chick type II collagens. J Immunol 149:309-316.

Harris JM and Chess RB (2003) Effect of pegylation on pharmaceuticals. Nat Rev Drug Discov 2:214-221. 
Helmick CG, Felson DT, Lawrence RC, Gabriel S, Hirsch R, Kwoh CK, Liang MH, Kremers HM, Mayes MD, Merkel PA, et al.; National Arthritis Data Workgroup (2008) Estimates of the prevalence of arthritis and other rheumatic conditions in the United States. Part I. Arthritis Rheum 58:15-25.

Hu X, Laragione T, Sun L, Koshy S, Jones KR, Ismailov II, Yotnda P, Horrigan FT, Gulko PS, and Beeton C (2012) KCa1.1 potassium channels regulate key proinflammatory and invasive properties of fibroblast-like synoviocytes in rheumatoid arthritis. J Biol Chem 287:4014-4022.

Imlach WL, Finch SC, Dunlop J, Meredith AL, Aldrich RW, and Dalziel JE (2008) The molecular mechanism of "ryegrass staggers," a neurological disorder of $\mathrm{K}+$ channels. $J$ Pharmacol Exp Ther 327:657-664.

Joundi RA, Brittain JS, Jenkinson N, Green AL, and Aziz T (2011) Rapid tremor frequency assessment with the iPhone accelerometer. Parkinsonism Relat Disord 17:288-290.

Kahlenberg JM and Fox DA (2011) Advances in the medical treatment of rheumatoid arthritis. Hand Clin 27:11-20.

Kostikis N, Hristu-Varsakelis D, Arnaoutoglou M, and Kotsavasiloglou C (2015) A smartphone-based tool for assessing parkinsonian hand tremor. IEEE J Biomed Health Inform 19:1835-1842.

Laragione T, Brenner M, Mello A, Symons M, and Gulko PS (2008) The arthritis severity locus Cia5d is a novel genetic regulator of the invasive properties of synovial fibroblasts. Arthritis Rheum 58:2296-2306.

Laragione T and Gulko PS (2010) mTOR regulates the invasive properties of synovial fibroblasts in rheumatoid arthritis. Mol Med 16:352-358.

Li W, Gao SB, Lv CX, Wu Y, Guo ZH, Ding JP, and Xu T (2007) Characterization of voltage-and $\mathrm{Ca} 2+$-activated $\mathrm{K}+$ channels in rat dorsal root ganglion neurons. $J$ Cell Physiol 212:348-357.

Lippiat JD, Standen NB, Harrow ID, Phillips SC, and Davies NW (2003) Properties of $\mathrm{BK}(\mathrm{Ca})$ channels formed by bicistronic expression of hSloalpha and beta1-4 subunits in HEK293 cells. J Membr Biol 192:141-148.

Löhn M, Lauterbach B, Haller H, Pongs O, Luft FC, and Gollasch M (2001) Beta(1) subunit of BK channels regulates arterial wall[Ca(2+)] and diameter in mouse cerebral arteries. J Appl Physiol (1985) 91:1350-1354.

Lundy SK, Sarkar S, Tesmer LA, and Fox DA (2007) Cells of the synovium in rheumatoid arthritis. T lymphocytes. Arthritis Res Ther 9:202.

Mannowetz N, Naidoo NM, Choo SA, Smith JF, and Lishko PV (2013) Slo1 is the principal potassium channel of human spermatozoa. eLife 2:e01009.

Meera P, Wallner M, and Toro L (2000) A neuronal beta subunit (KCNMB4) makes the large conductance, voltage- and $\mathrm{Ca} 2+$-activated $\mathrm{K}+$ channel resistant to charybdotoxin and iberiotoxin. Proc Natl Acad Sci USA 97:5562-5567.

Meredith AL, Thorneloe KS, Werner ME, Nelson MT, and Aldrich RW (2004) Overactive bladder and incontinence in the absence of the BK large conductance Ca2+-activated K+ channel. J Biol Chem 279:36746-36752.

Miyazaki H, Oyama F, Inoue R, Aosaki T, Abe T, Kiyonari H, Kino Y, Kurosawa M, Shimizu J, Ogiwara I, et al. (2014) Singular localization of sodium channel $\beta 4$ subunit in unmyelinated fibres and its role in the striatum. Nat Commun 5:5525. Mobasheri A, Lewis R, Ferreira-Mendes A, Rufino A, Dart C, and Barrett-Jolley R (2012) Potassium channels in articular chondrocytes. Channels (Austin) 6:416-425

Otero M and Goldring MB (2007) Cells of the synovium in rheumatoid arthritis. Chondrocytes. Arthritis Res Ther 9:220.

Pethő Z, Tanner MR, Tajhya RB, Huq R, Laragione T, Panyi G, Gulko PS, and Beeton C (2016) Different expression of $\beta$ subunits of the KCa1.1 channel by invasive and non-invasive human fibroblast-like synoviocytes. Arthritis Res Ther 18:103.

Poulsen AN, Wulf H, Hay-Schmidt A, Jansen-Olesen I, Olesen J, and Klaerke DA (2009) Differential expression of BK channel isoforms and beta-subunits in rat neuro-vascular tissues. Biochim Biophys Acta 1788:380-389.

Rosengren S, Boyle DL, and Firestein GS (2007) Acquisition, culture, and phenotyping of synovial fibroblasts. Methods Mol Med 135:365-375.
Sanchez M and McManus OB (1996) Paxilline inhibition of the alpha-subunit of the high-conductance calcium-activated potassium channel. Neuropharmacology 35: 963-968.

Schett G (2007) Cells of the synovium in rheumatoid arthritis. Osteoclasts. Arthritis Res Ther 9:203.

Shih YH and Young MS (2007) Integrated digital image and accelerometer measurements of rat locomotor and vibratory behaviour. $J$ Neurosci Methods 166: $81-88$

Tajhya RB, Hu X, Tanner MR, Huq R, Kongchan N, Neilson JR, Rodney GG, Horrigan FT, Timchenko LT, and Beeton C (2016) Functional KCa1.1 channels are crucial for regulating the proliferation, migration and differentiation of human primary skeletal myoblasts. Cell Death Dis 7:e2426.

Tanner MR and Beeton C (2018) Differences in ion channel phenotype and function between humans and animal models. Front Biosci 23:43-64.

Tanner MR, Hu X, Huq R, Tajhya RB, Sun L, Khan FS, Laragione T, Horrigan FT, Gulko PS, and Beeton C (2015) KCa1.1 inhibition attenuates fibroblast-like synoviocyte invasiveness and ameliorates disease in rat models of rheumatoid arthritis. Arthritis Rheumatol 67:96-106.

Tanner MR, Pennington MW, Laragione T, Gulko PS, and Beeton C (2017a) KCa1.1 channels regulate $\beta_{1}$-integrin function and cell adhesion in rheumatoid arthritis fibroblast-like synoviocytes. FASEB $J$ 31:3309-3320.

Tanner MR, Tajhya RB, Huq R, Gehrmann EJ, Rodarte KE, Atik MA, Norton RS, Pennington MW, and Beeton C (2017b) Prolonged immunomodulation in inflammatory arthritis using the selective Kv1.3 channel blocker HsTX1[R14A] and its PEGylated analog. Clin Immunol 180:45-57.

Tarcha EJ, Chi V, Muñoz-Elías EJ, Bailey D, Londono LM, Upadhyay SK, Norton K, Banks A, Tjong I, Nguyen H, et al. (2012) Durable pharmacological responses from the peptide ShK-186, a specific Kv1.3 channel inhibitor that suppresses T cell mediators of autoimmune disease. J Pharmacol Exp Ther 342:642-653.

Tauc M, Congar P, Poncet V, Merot J, Vita C, and Poujeol P (1993) Toxin pharmacology of the large-conductance $\mathrm{Ca}(2+)$-activated $\mathrm{K}+$ channel in the apical membrane of rabbit proximal convoluted tubule in primary culture. Pflugers Arch $\mathbf{4 2 5}$ : 126-133.

Tolboom TC, van der Helm-Van Mil AH, Nelissen RG, Breedveld FC, Toes RE and Huizinga TW (2005) Invasiveness of fibroblast-like synoviocytes is an individual patient characteristic associated with the rate of joint destruction in patients with rheumatoid arthritis. Arthritis Rheum 52:1999-2002.

Turecek PL, Bossard MJ, Schoetens F, and Ivens IA (2016) PEGylation of biopharmaceuticals: a review of chemistry and nonclinical safety information of approved drugs. J Pharm Sci 105:460-475.

Uebele VN, Lagrutta A, Wade T, Figueroa DJ, Liu Y, McKenna E, Austin CP, Bennett PB, and Swanson R (2000) Cloning and functional expression of two families of $\beta$-subunits of the large conductance calcium-activated $\mathrm{K}+$ channel. $J$ Biol Chem 275:23211-23218.

Wang B, Jaffe DB, and Brenner R (2014) Current understanding of iberiotoxinresistant BK channels in the nervous system. Front Physiol 5:382.

Weidema AF, Dixon SJ, and Sims SM (2000) Electrophysiological characterization of ion channels in osteoclasts isolated from human deciduous teeth. Bone 27:5-11.

Yu M, Liu SL, Sun PB, Pan H, Tian CL, and Zhang LH (2016) Peptide toxins and small-molecule blockers of BK channels. Acta Pharmacol Sin 37:56-66.

Address correspondence to: Dr. Christine Beeton, Biology of Inflammation Center and Center for Drug Discovery, Baylor College of Medicine, One Baylor Plaza, Mail Stop BCM335, Room S409A, Houston, TX 77030. E-mail: beeton@ bcm.edu 